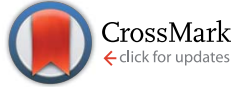

Cite this: RSC Adv., 2017, 7, 7293

Received 3rd January 2017

Accepted 12th January 2017

DOI: $10.1039 / c 7 r a 00052 a$

www.rsc.org/advances

\section{Toward high-performance poly(para-phenylene terephthalamide) (PPTA)-based composite paper via hot-pressing: the key role of partial fibrillation and surface activation}

\author{
Zhaoqing Lu, ${ }^{\star a b}$ Wanbin Dang, ${ }^{a}$ Yongsheng Zhao, ${ }^{* a}$ Lamei Wang, ${ }^{a}$ Meiyun Zhang ${ }^{a}$ \\ and Guodong Liu ${ }^{a}$
}

The inert surface of para-aramid fibers makes para-aramid fiber-based composites suffer from a poor interfacial interaction and limited physical properties. In this work, the influence of hot-pressing on paper structural properties was explored. Without hot-pressing, in comparison with the fibrid paper (Paper-I), the addition of para-aramid chopped-fiber obviously enhanced mechanical properties, but led to slight damage of the dielectric properties of the fiber/fibrid paper (Paper-II) because of voids and defects. After hot-pressing, it was noteworthy that the increased mechanical properties and dielectric properties of the papers were mainly due to the individual pressing treatment and independent of the individual heating treatment. Interestingly, the combined treatment of heating and pressing shows a synergistic effect and results in an increase of paper compactness especially for Paper-II. Meanwhile, the interfacial interaction between different fibers in the composite papers was greatly improved, which is mainly associated with the partial dissociation into a fibrillar structure and the improved surface activation of para-aramid chopped fibers. Therefore, fiber micro-fibrillation is highly effective for improving internal bonding and optimizing physical properties for para-aramid-based composite papers.

\section{Introduction}

Polymer-based composites have gained great interest from both the academic community and industrial engineers due to their easy processing and multifunctional properties. ${ }^{1-3}$ Poly(paraphenylene terephthalamide) (PPTA)-based papers have also achieved extensive applications; they are mostly used as insulation materials and honeycomb-structured materials, ${ }^{4}$ covering aerospace technologies, ${ }^{5-7}$ telecommunication, transportation and even weapon manufacturing owing to their exceptional properties including high mechanical strength, good heat resistance, and favorable insulation properties. ${ }^{8-15}$ In general, conventional aramid paper consists of aramid pulp and chopped fibers and is popular in many high value-added applications. ${ }^{\mathbf{1 6 - 1 8}}$

PPTA fibrid, a newly-developed special product of aramid fibers, is generally manufactured through a precipitation process by applying high shear on the aramid solution in its non-solvent. ${ }^{19,20}$ PPTA fibrid thus inherits many advantages such as high mechanical strength, good heat resistance and

${ }^{a}$ College of Bioresources Chemical and Materials Engineering, Shaanxi University of Science \& Technology, Xi'an, 710021, China. E-mail: zhaoyongsheng@sust.edu.cn; Tel: $+86-135-7292-7693$

${ }^{b}$ State Key Laboratory of Pulp and Paper Engineering, South China University of Technology, Guangzhou, 510640, China favorable papermaking property. ${ }^{21}$ Due to its large specific surface area, better flexibility, and dispersion stability in water, PPTA fibrid is suitable for making PPTA paper-based composite and can be used to replace the PPTA pulp. ${ }^{21}$ Indeed, our previous work demonstrates a successful property optimization including improved papermaking property and enhancement in mechanical strength for aramid chopped fiber/fibrid composite paper in comparison with conventional aramid chopped fiber/pulp composite paper. ${ }^{22,23}$ However, the aramid fiber/fibrid composite paper still needs further optimization in paper properties including mechanical properties and insulation properties to extend its applications. ${ }^{24-26}$

Since the chopped fiber surface is smooth and has less chemical reactive groups, changing the inert surface and improving surface activity of the aramid chopped fiber is of great importance. ${ }^{\mathbf{2 7 - 2 9}}$ Previous reports have made great efforts including chemical etching by mixed acids, ${ }^{30}$ ionization, surface irradiation by plasma, ${ }^{31-34}$ ultrasonic treatment, chemical grafting methods, ${ }^{35-37}$ and fluorinated modification of the molecular chains ${ }^{38}$ and other approaches..$^{35,39-41}$ For instance, phosphoric acid treatment on PPTA showed increasing content of surface functional groups but resulted in a declined mechanical property of PPTA fibers. ${ }^{42-44}$ Gas plasma treatment on Kevlar fibers caused remarkably improved interfacial shear strength (IFSS) between the fiber and epoxy resin during pull- 
out tests. ${ }^{31}$ But the densities of active groups in resultant fibers are still not homogeneous or rich. Recently, Liu and his coworkers have realized direct fluorination of para-aramid fibers which led to surface chain cleavage behavior and even covalent modification on the surface of aramid fibers. ${ }^{\mathbf{4 1}}$ The direct fluorination process needs complex reaction conditions. ${ }^{45-47}$ In a word, some approaches cannot directly achieve surface activation in high-efficiency or high density of functional groups without sacrificing the high property or integrity of chemical structure in PPTA fiber, while the others needs special reaction technology and can only be achieved under harsh conditions. ${ }^{\mathbf{4 8 , 4 9}}$ Besides, hot-pressing under appropriate condition has been also demonstrated to significantly enhance the mechanical properties as well as the insulation properties of PPTA-based papers. Obviously, it is an easy and convenient method for modification of PPTA-based papers in comparison with the aforementioned approaches. In addition, hot-pressing also induces some morphological changes for para-aramid fibers and para-aramid fibrids. Unluckily, the reinforcing mechanism of hot-pressing is not clearly elucidated.

Hence, the objective of this work was to systematically investigate the effect of post-treatment through hot-pressing on paper structural properties. For this purpose, two different modes, namely, individual heating or pressing treatment and combined heating/pressing treatment were applied during hotpressing. The important properties including mechanical properties and insulation properties were discussed. At the same time, we also focused on the reinforcing mechanisms and paper structural changes of para-aramid fiber-based papers with the aid of hot-pressing. Hopefully, the results can give a deep insight into the structure-property relationship of PPTAbased papers during hot-pressing and enrich the knowledge of reinforcing mechanisms for PPTA-based papers.

\section{Experimental}

\subsection{Materials and papermaking process}

para-Aramid chopped fiber with average diameters of $12 \mu \mathrm{m}$ and length of 4-5 $\mathrm{mm}$ was purchased from Hebei Guigu
Chemical company. para-Aramid fibrid was provided by Teijin Company. The aramid chopped fiber/fibrid composite paper was prepared according to TAPPI standard T 205 sp-95. ${ }^{50,51}$ As shown in Fig. 1, firstly, to prepare the uniform pulp suspension at $0.1 \%$ consistency, a mixture of aramid chopped fiber and fibrid at the mass ratio of $7: 3$ was disintegrated in water via a standard disperser. Then, the aramid chopped fiber/fibrid composite paper was prepared by an ERNST HAAGEBBS-3 sheet formation machine, and the target grammage of produced sample was set as $45 \mathrm{~g} \mathrm{~m}^{-2}$. To obtain dried chopped fiber/fibrid composite paper, the formed wet-sample was pressed at $0.04 \mathrm{MPa}$ for $3 \mathrm{~min}$ and then was dried at $105^{\circ} \mathrm{C}$ for 5 min. Subsequently, a hot-pressing machine was employed for post-treating on the composite papers. For comparison, paraaramid fibrid paper was also prepared under the same condition as a reference sample. For simplicity, the reference sample was named as Paper-I and the composite paper was named as Paper-II.

\subsection{Characterization}

The mechanical properties of PPTA-based papers were determined via the measurement of tensile index and tearing index by a L\&W SE-062 tensile strength tester and a TMI tearing strength tester according to TAPPI standard T 494 om-88 and T411 om-97, respectively. Before that, the specimens for tensile testing were cut into a rectangular shape, which possesses dimensions of $15 \times 100 \mathrm{~mm}^{2}$ with a thickness of $\sim 0.06 \mathrm{~mm}$. To evaluate the insulation property of aramid chopped fiber/fibrid composite paper, the measurement of electrical breakdown strength was performed on a CS2672D dielectric strength tester according to ASTM D149 standard.

The surface morphologies of aramid chopped fiber/fibrid composite paper with and without post-treatment were characterized by $\mathrm{S}-4800$ field emission scanning electron microscope (SEM) (Hitachi, Japan) with the operation voltage of $3 \mathrm{kV}$. Prior to analysis, produced samples were coated with the platinum layer to provide the conductivity for impinging electrons. A LEICA super depth field microscope (Germany) was used to

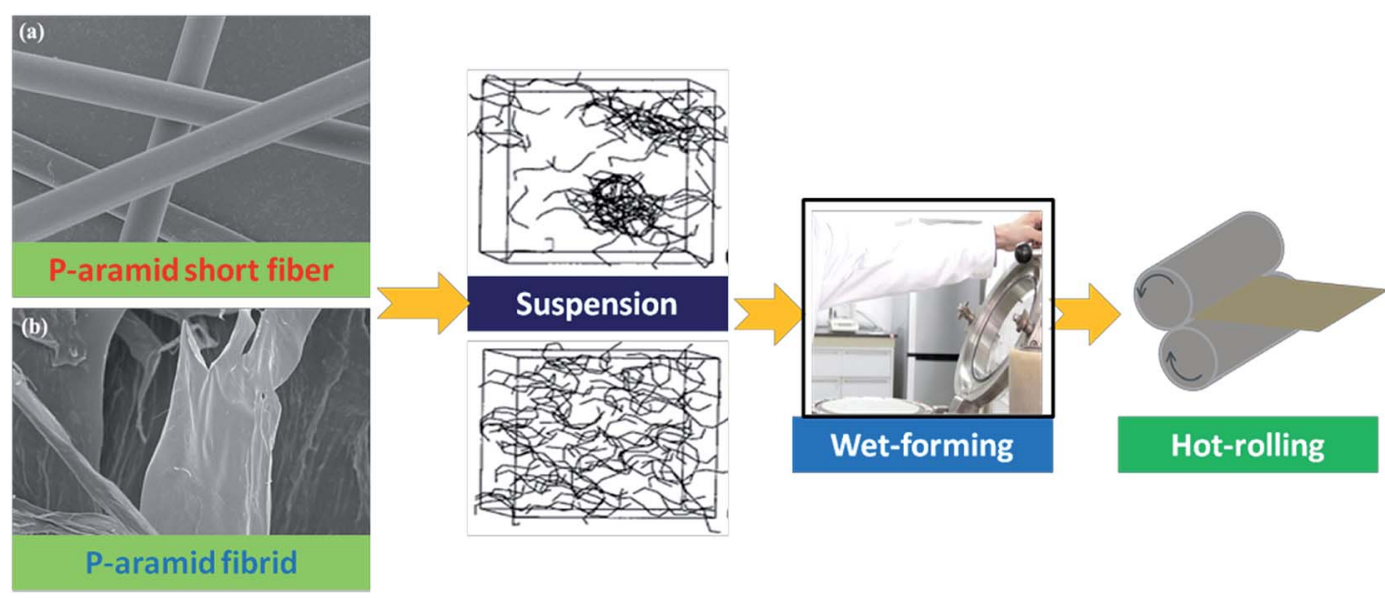

Fig. 1 Sketch of the papermaking process and post-treatment with the aid of hot pressing for PPTA-based papers. 
observe the fracture surface of original and post-treated PPTAbased papers.

X-ray Photoelectron Spectroscopy (XPS) analysis was carried out using a Kratos ASMA 800 spectrometer (Kratos Analytical Ltd., UK) equipped with Al Ka X-ray source. During the test, the $\mathrm{X}$-ray operation power as $450 \mathrm{~W}$ and the vacuum chamber pressure was maintained at the range of $10^{-8}$ to $10^{-9}$ Torr.

\section{Results and discussion}

\subsection{The influence of heating and pressing on physical property of PPTA-based papers}

In order to explore the key parameter in hot-pressing, the effect of individual heating treatment or individual pressing treatment on the mechanical properties and insulation properties were investigated. Fig. 2 shows the representative mechanical properties and insulation properties of two kinds of aramid fiber paper when solely treated either by heating at different temperatures or applying different pressure. Firstly, it can be found that individual heating or pressing treatment can significantly enhance the tensile strength and the tearing strength of the composite paper in comparison with Paper-I. The insulation property of Paper-II is lower than that of Paper-I which is mainly due to defects and decreased paper compactness caused by the introduction of chopped fibers. With changing heating temperature, the insulation property of both Paper-I and Paper-II remains unchanged. Hence, to achieve an overall property, emphasis should be taken on the balance between the mechanical property enhancement and insulation property deduction by using appropriate temperature and/or pressure during hot-pressing. Secondly, the mechanical property and insulation property of Paper-I and
Paper-II were compared when concerning temperature increment or pressure increment. It was found that individual heating treatment is indeed in favor of tensile property enhancement, i.e., increasing from $\sim 15 \mathrm{MPa}$ to $\sim 35 \mathrm{MPa}$. But with increasing temperature, it is less effective to further enhance the tensile strength of Paper-I and Paper-II. However, when increasing pressure especially at high pressure value $(>0.3$ $\mathrm{MPa}$ ), the tensile strength further increases to a high level of $\sim 45 \mathrm{MPa}$ while the tearing strength of Paper-II decreases to $\sim 18 \mathrm{MPa}$ which is still comparable with the value of only heating-treated paper. Interestingly, applying pressure can enhance insulation property remarkably though Paper-II shows a weaker insulation property than Paper-I. In addition, with increasing pressure, the insulation property can be further increased. Overall, the mechanical property improvement and depression of insulation property for Paper-II is insensitive to temperature variation during heating treatment. High pressure along with constant thermal condition is potentially favorable for enhancing the tensile strength and electrical property, that is, a balanced property. It can be concluded that the increased mechanical properties and dielectric properties of both Paper-I and Paper-II were dominated by individual pressing treatment but independent on individual heating treatment.

For a clear comparison, the combined effect of heat and pressure on the mechanical properties and insulation properties of these papers were further investigated. As shown in Fig. 3, two different modes were implemented. The first case is that high pressure is considered as a basic condition and the effect of temperature variation at a fixed pressure of $0.4 \mathrm{MPa}$ was investigated. The tensile strength and dielectric strength of Paper-II begin to be sensitive to temperature variation. With increasing temperature from $40{ }^{\circ} \mathrm{C}$ to $260{ }^{\circ} \mathrm{C}$, the tensile

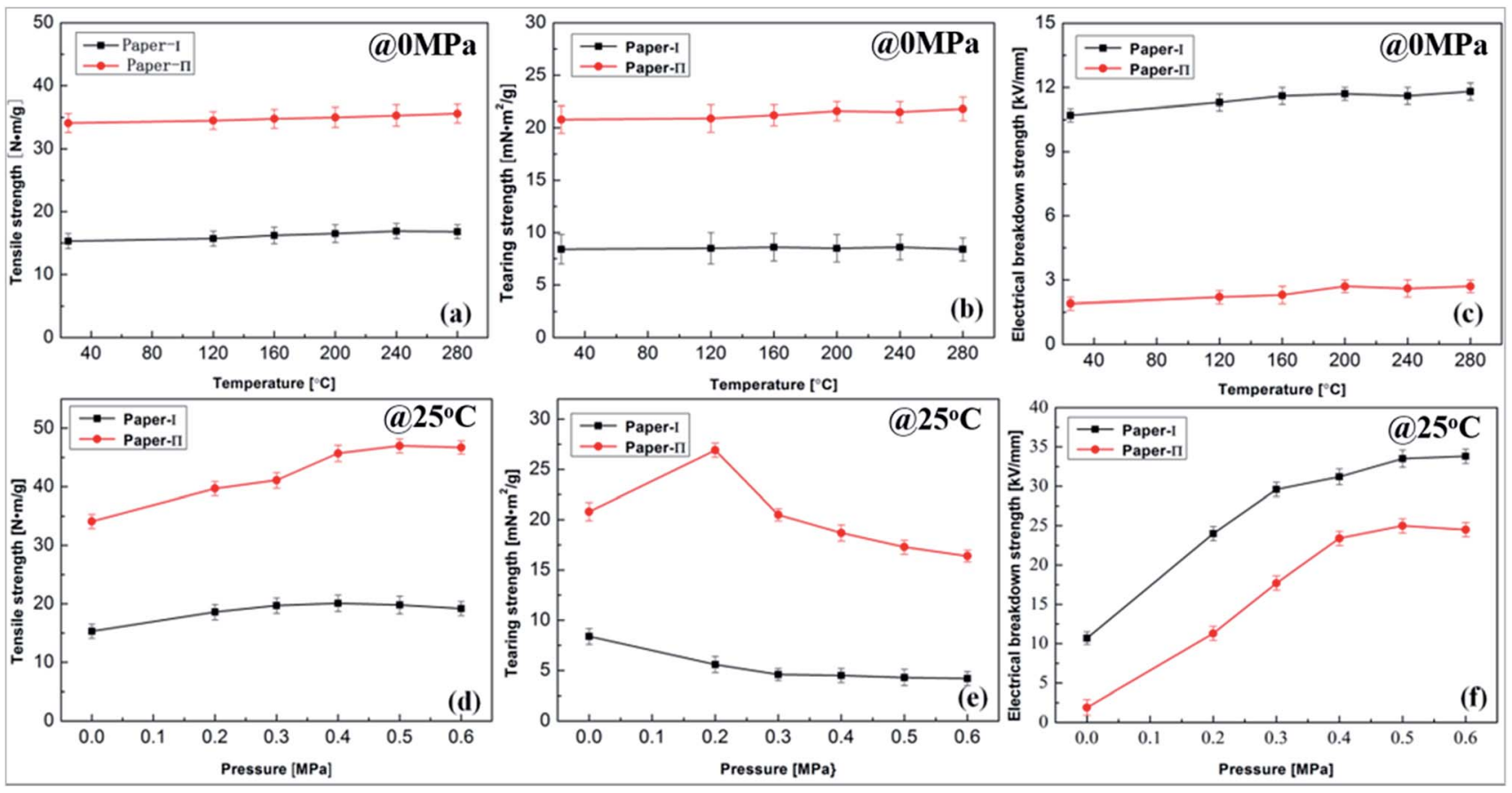

Fig. 2 The effect of individual heating and pressing treatment on the mechanical properties and insulation properties of PPTA-based papers. 

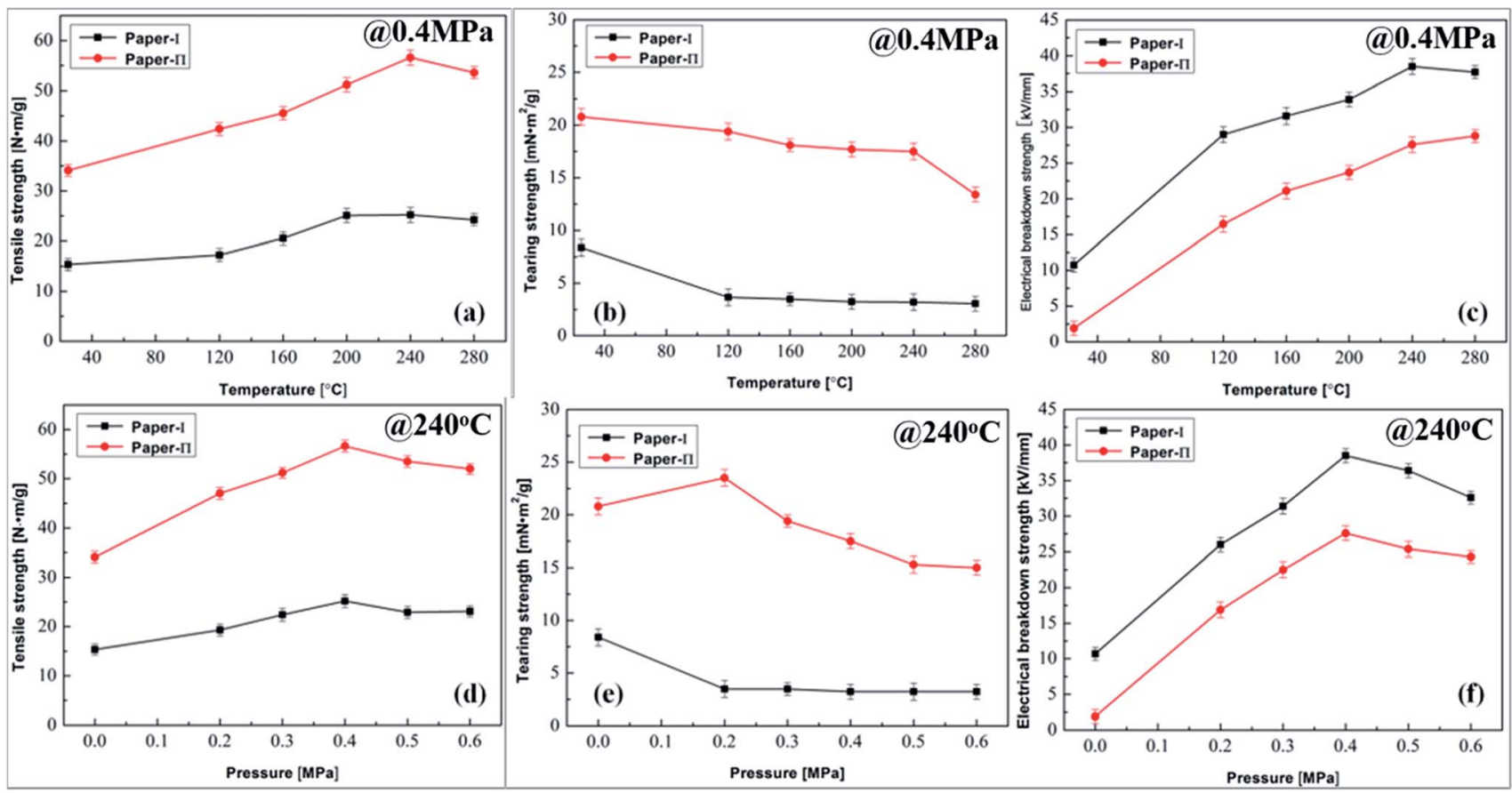

Fig. 3 The combined heating and pressing treatment on the mechanical properties and insulation properties of PPTA-based papers.

strength of Paper-II gradually increases to $\sim 55 \mathrm{MPa}$ and the dielectric strength of Paper-II also increases from $2 \mathrm{kV} \mathrm{mm}^{-1}$ to $27 \mathrm{kV} \mathrm{mm}{ }^{-1}$. It is noteworthy that the tensile strength and dielectric strength of Paper-II treated by heating at fixed pressure are larger than those of Paper-II treated by individual heating or pressing, showing a great synergistic effect. In addition, the tensile strength and dielectric strength of Paper-I is also enhanced under the same treating condition. The second case is to change pressure at a fixed temperature of $240{ }^{\circ} \mathrm{C}$. Similarly, a further property enhancement was also achieved, that is, Paper-II with tensile strength of $\sim 55 \mathrm{MPa}$ and dielectric strength of $\sim 25 \mathrm{kV} \mathrm{mm}{ }^{-1}$ and Paper-I with tensile strength of $\sim 22 \mathrm{MPa}$ and dielectric strength of $\sim 37 \mathrm{~V} \mathrm{~mm}^{-1}$. Though a slight decrease of the tearing strength and the dielectric strength for these PPTA-based papers were observed especially at high pressure, a synergistic effect of heating and pressing on property enhancement of papers was also obvious when changing pressure at fixed temperature.

To present a clear evidence of synergistic effect of heating and pressing, the best performance of Paper-I and Paper-II obtained under different conditions were summarized and compared as can be seen from Fig. 4. For Paper-I, it can be found that it achieves a further increase of $27 \%$ in tensile strength and $15 \%$ in dielectric strength as well as a comparable tearing strength when treated by combined heating and pressing in comparison with individual pressing-treated case. Correspondingly, Paper-II also achieves a further increase in tensile strength and dielectric strength as well as similar tearing resistance in a same way. Overall, through combined heating and pressing treatment, the synergistic effect endows Paper-II a superhigh performance with tensile strength of $56.6 \mathrm{MPa}$, tearing strength of $15.0 \mathrm{MPa}$ and dielectric strength of $27.6 \mathrm{kV}$ $\mathrm{mm}^{-1}$, namely a comprehensive increase in comparison with those of Paper-I.

\subsection{The influence of hot pressing on paper structures in PPTA-based papers}

In the pursuit of high-performance PPTA-based papers, previous experimental and theoretical investigations have pointed out that the fiber-fiber joint and fiber-fibrid interactions play key roles in enhancing paper performance. In order to investigate the effect of hot-pressing process on aramid-based paper structures, morphological changes, fracture surfaces and internal bonding strength of papers after post-treatment via hot-pressing were systematically analyzed. Fig. 5 presents morphological details in Paper-I (control sample) before and after post-treatment. Post-treatment is in great favor for physical connections between different fibrids as can be seen that the surface became smooth and defects were greatly improved.

Fig. 6 shows morphological details in Paper-II before and after post-treatment. Before post-treatment, Paper-II presents rough surface and porous structure which indicates a looselypacked network structure with large pores. In addition, the aramid chopped fiber shows a smooth surface and aramid fibrid retains its pristine flexible morphology. Fig. 6a shows several patterns for aramid chopped fibers and aramid fibrids. The aramid fibrid are inclined to deposit on the chopped fiber while the introduction of aramid chopped fiber destructs joint effect between different fibrids, leading to a large quantity of pores and defects in the paper.

Hence, mechanical performance of Paper-II is strongly depending on the interaction between aramid chopped fiber and aramid fibrid. In this case, Paper-II shows randomly 

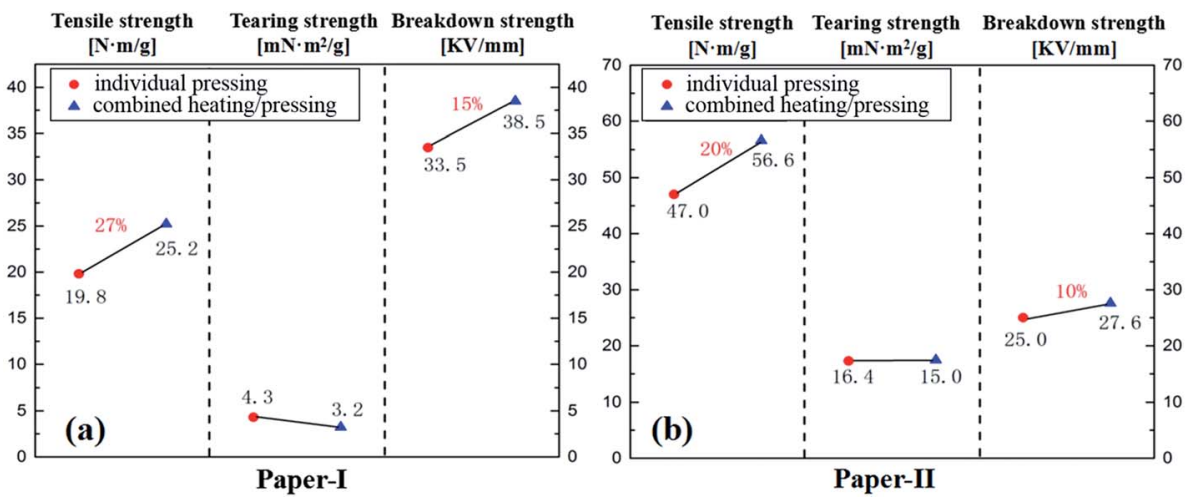

Fig. 4 Summary of the best performance for PPTA-based paper obtained under different treating conditions: (a) Paper-I; (b) Paper-II.
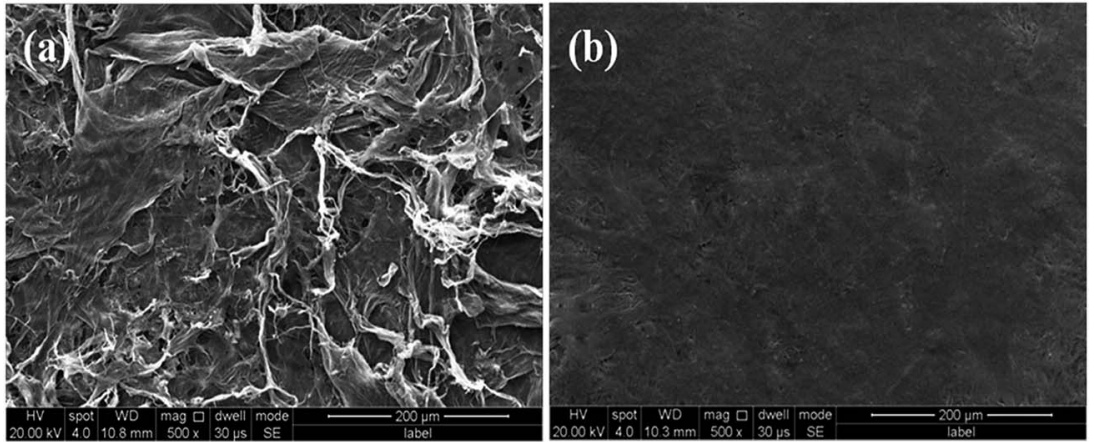

Fig. 5 Morphological changes of Paper-I during heating/pressing treatment: (a) before post-treatment; (b) after post-treatment.

overlapped short fiber along with some fiber surrounded by aramid fibrid mainly due to physical adhesion. After posttreatment as can be seen in Fig. 6b, the treated paper achieves smooth surface with decreased pore size, resulting in increased paper compactness. In addition, the deformed aramid chopped fiber is in favor of interconnection between aramid chopped fiber and aramid fibrid. The aramid fibrid is inclined to wrap closely on the surface of aramid chopped fibers. Therefore, post-treatment favors paper compactness and the interfacial adhesion between aramid chopped fiber and aramid fibrid.

Subsequently, fracture surface of aramid-based papers was examined to roughly estimate interfacial adhesion strength. Optical images for fracture surfaces of these papers can be found in Fig. 7. It was found that the chopped fibers are pulled out easily for Paper-II without post-treatment, while fiber fracture occurs for Paper-II after post-treatment. It indicates greatly enhanced interfacial adhesion strength because of post-

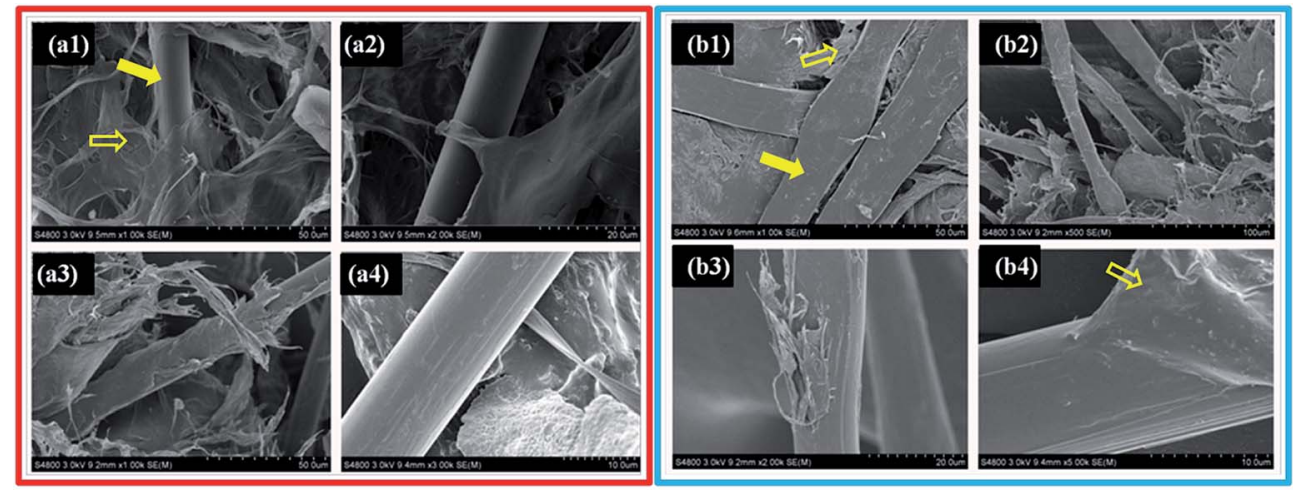

Fig. 6 Morphological changes of Paper-II during the combined heating/pressing treatment: (a1-a4) before post-treatment; and (b1-b4) after post-treatment. Note: solid arrow indicates fibers while hollow arrow represents fibrid. 

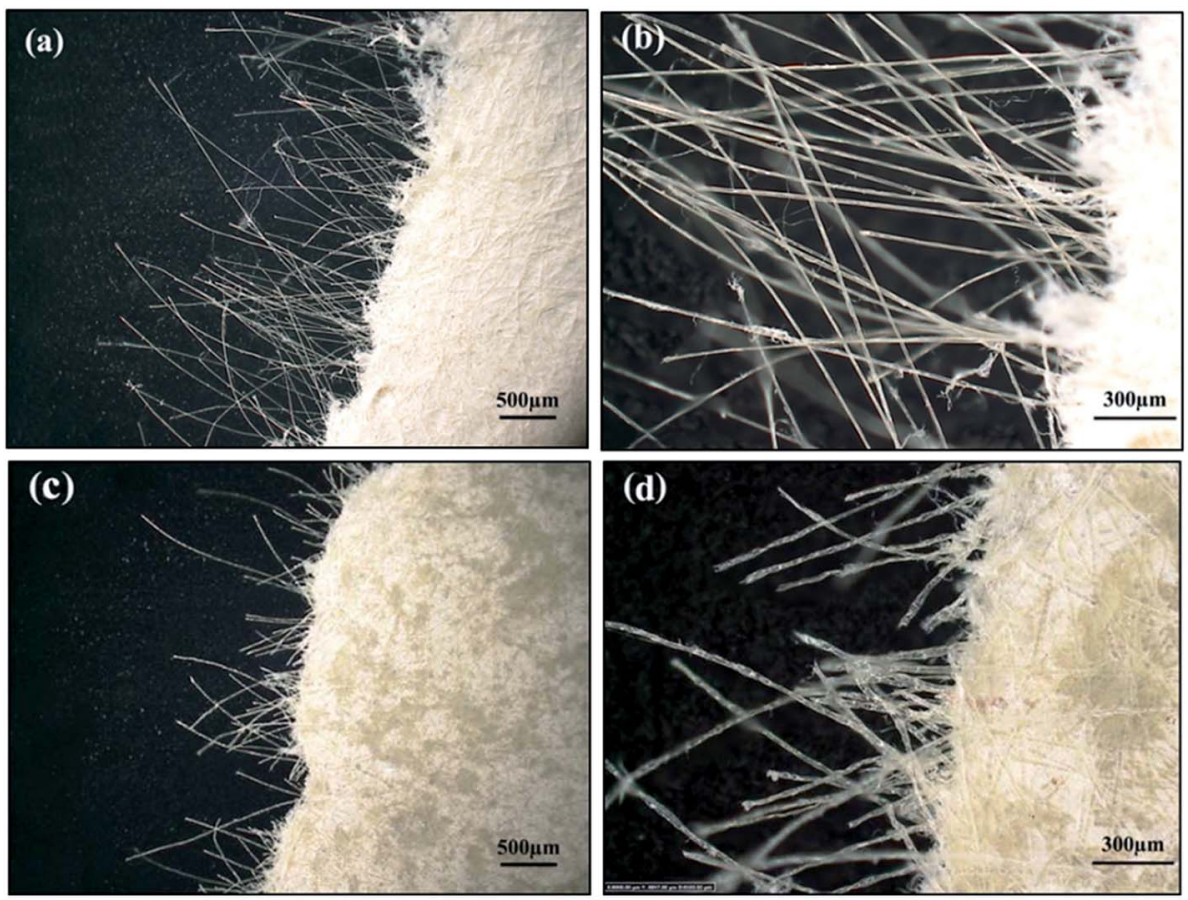

Fig. 7 The fracture surface of Paper-II during the combined heating/pressing treatment: (a) and (b) without post-treatment; (c) and (d) after post-treatment.

treatment, which is advantageous for stress transferring and paper's final performance.

In order to quantitatively analyze the effect of the combined heating/pressing treatment on internal adhesion, "Scott interlayer bonding strength" was used. Fig. 8 presents interlayer bonding strength of PPTA-based papers as a function of temperature or pressure. In comparison with Paper-I, the introduction of aramid chopped fiber decreases the interlayer bonding strength. As shown in Fig. 8a, with increasing temperature at fixed pressure of 0.4 $\mathrm{MPa}$, interlayer bonding strength of both Paper-I and Paper-II continuously increases to a high level. When increasing pressure at fixed temperature of $240{ }^{\circ} \mathrm{C}$, interlayer bonding strength increase slowly at low pressure but increasing abruptly when the pressure reaches $0.3 \mathrm{MPa}$. And the interlayer bonding strength gradually reaches to a plateau when further increase pressure to 0.6 MPa. Overall, the interlayer bonding strength is indeed has positive effect on the resultant mechanical properties of aramid fibers. At the same time, the results indicate the possibility of microstructure and chemical changes of chopped fibers and fibrids induced by hot-pressing.

\subsection{The influence of hot-pressing on chemical structure and microstructures of aramid choped fiber and aramid fibrid}

XPS can be used to evaluate the chemical structural changes of aramid chopped fiber and aramid fibrid after the combined
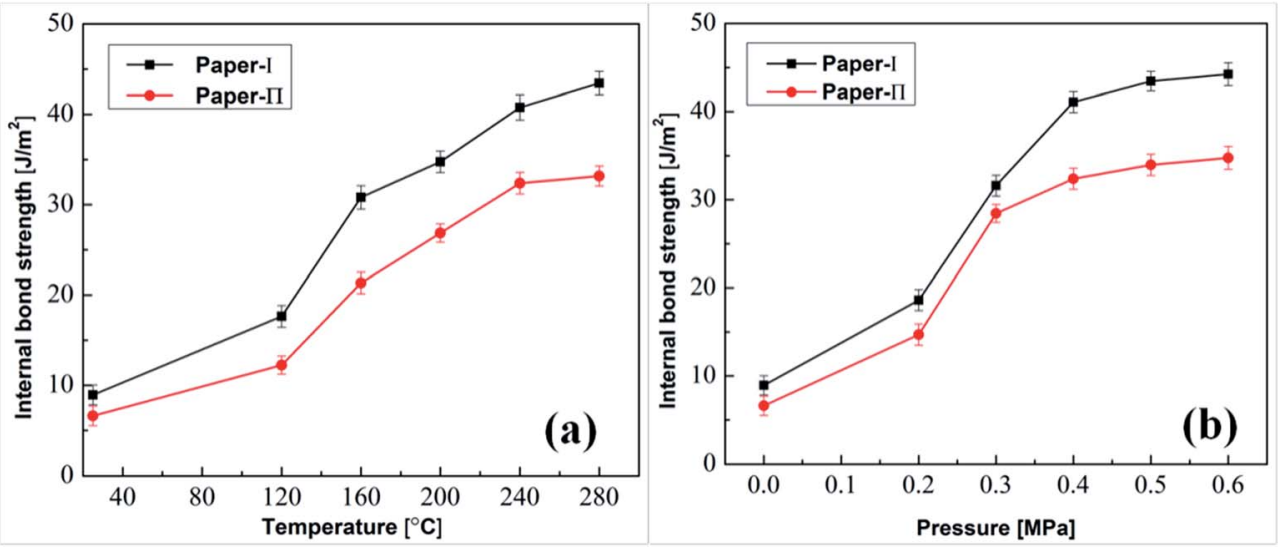

Fig. 8 Interlayer bonding strength of Paper-I and Paper-II under two different treatment conditions: (a) as a function of treatment temperature at a fixed pressure of $0.4 \mathrm{MPa}$; (b) as a function of treatment pressure at a fixed temperature of $240{ }^{\circ} \mathrm{C}$. 
heating/pressing treatment. For aramid chopped fiber without treatment (Fig. 9a), there are three peaks indicating $-\mathrm{C}-\mathrm{C}-$ group $(284.9 \mathrm{eV}),-\mathrm{C}-\mathrm{N}-$ group $(286.9 \mathrm{eV})$ and $-\mathrm{C}=\mathrm{O}$ group (290.6 eV), respectively. After heating/pressing treatment, a new peak emerges, representing the existence of -C-O- group (287.8 $\mathrm{eV}$ ). As can be seen in Table 1, the ratio for oxygen-containing groups gradually increases from $7.17 \%$ to $31.87 \%$ while the ratio for -C-C- group decreases from $68.61 \%$ to $30.76 \%$ after heat/pressure treatment. This is mainly attributed to that intramolecular bonding (hydrogen bonding) in aramid chopped fiber is destructed and finally activates the inert surface of aramid chopped fiber. For aramid fibrid, it changes in a totally different way. Before heating/pressing treatment (Fig. 9c), aramid fibrid possesses four different peaks representing $-\mathrm{C}-\mathrm{C}-$ group (284.6 eV), -C-N- group (285.9 eV), -C-O- group (287.7 $\mathrm{eV})$ and $-\mathrm{C}=\mathrm{O}(288.8 \mathrm{eV})$, respectively. After heating/pressing treatment on aramid fibrid (Fig. 9d), the peak for $-\mathrm{C}-\mathrm{O}-$ group disappears. Seen from Table 1 , the ratio for $-\mathrm{C}=\mathrm{O}$ group increases from $11.06 \%$ to $17.56 \%$. The increasing number of $-\mathrm{C}=\mathrm{O}$ group means potential hydrogen-bonding interaction with aramid chopped fiber. Hence, heating/pressing treatment can increase surface activation of aramid chopped fiber and aramid fibrid and thus increasing their interfacial adhesion strength.

\subsection{The mechanism for the realization of high performance PPTA-based paper via hot-pressing}

In order to further explore the reason for mechanical property enhancement in PPTA-based papers, the morphological
Table 1 Surface functional groups of aramid chopped fiber and aramid fibrid before and after the combined heating/pressing treatment

\begin{tabular}{llllr}
\hline & \multicolumn{4}{l}{ The ratio for functional groups [\%] } \\
\cline { 2 - 5 } & $-\mathrm{C}-\mathrm{C}-$ & $-\mathrm{C}-\mathrm{N}-$ & $-\mathrm{C}-\mathrm{O}-$ & $-\mathrm{C}=\mathrm{O}$ \\
\hline Fiber-before & 68.61 & 24.22 & - & 7.17 \\
Fiber-after & 30.76 & 37.37 & 22.30 & 9.57 \\
Fibrid-before & 43.13 & 39.58 & 6.22 & 11.06 \\
Fibrid-after & 42.90 & 39.54 & - & 17.56 \\
\hline
\end{tabular}

changes of aramid chopped fiber in related with pressure increment during the combined heating/pressing treatment were presented in Fig. 10. Relying on the intermolecular hydrogen-bonding interaction, molecular chains of PPTA can pack in a same crystalline plane. Due to the weak van der Waals force, lateral pressure can destruct the fiber structure and forming fibrillar structure. ${ }^{\mathbf{1 6 , 5 2 , 5 3}}$ For original aramid chopped fiber, it shows a uniform cylindrical form with smooth surface. As mentioned above, Paper-II is strongly sensitive to the pressure especially during the combined heating/pressing treatment. At low pressure, the aramid chopped fiber shows great deformation and becomes ribbon-like films, indicating a flattening process. When the pressure further increases, the aramid chopped fiber starts to crack and gives rise to a large quantity of fibrils, which is a partial dissociation process. At high pressure (such as $0.6 \mathrm{MPa}$ ), it can be seen that some fibrils become separated with ultrahigh aspect ratio, i.e., a partial
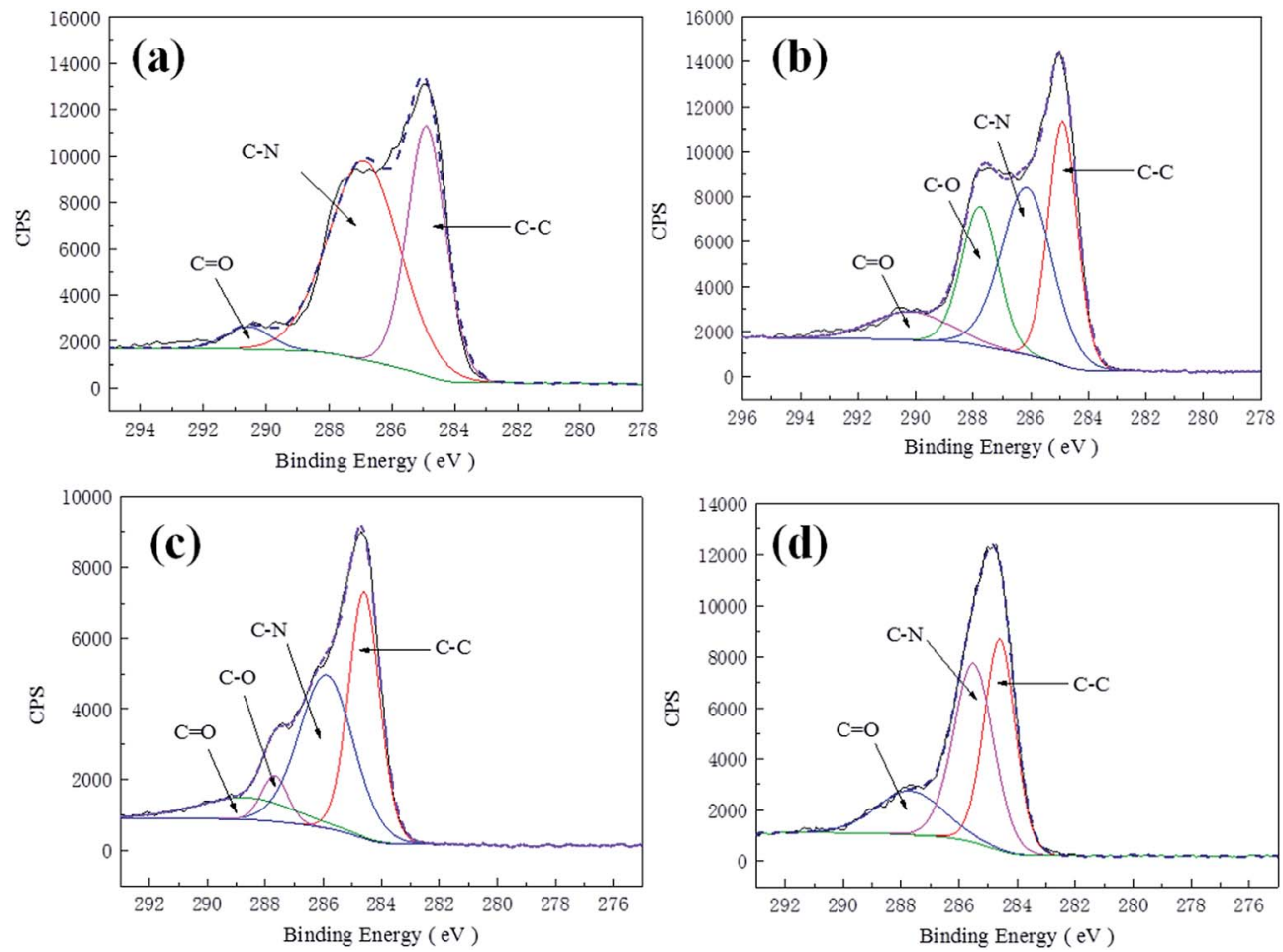

Fig. 9 XPS spectra of aramid chopped fiber and aramid fibrid. (a) Chopped fiber before hot-pressing; (b) chopped fiber after hot-pressing; (c) fibrid before hot-pressing; and (d) fibrid after hot-pressing. 

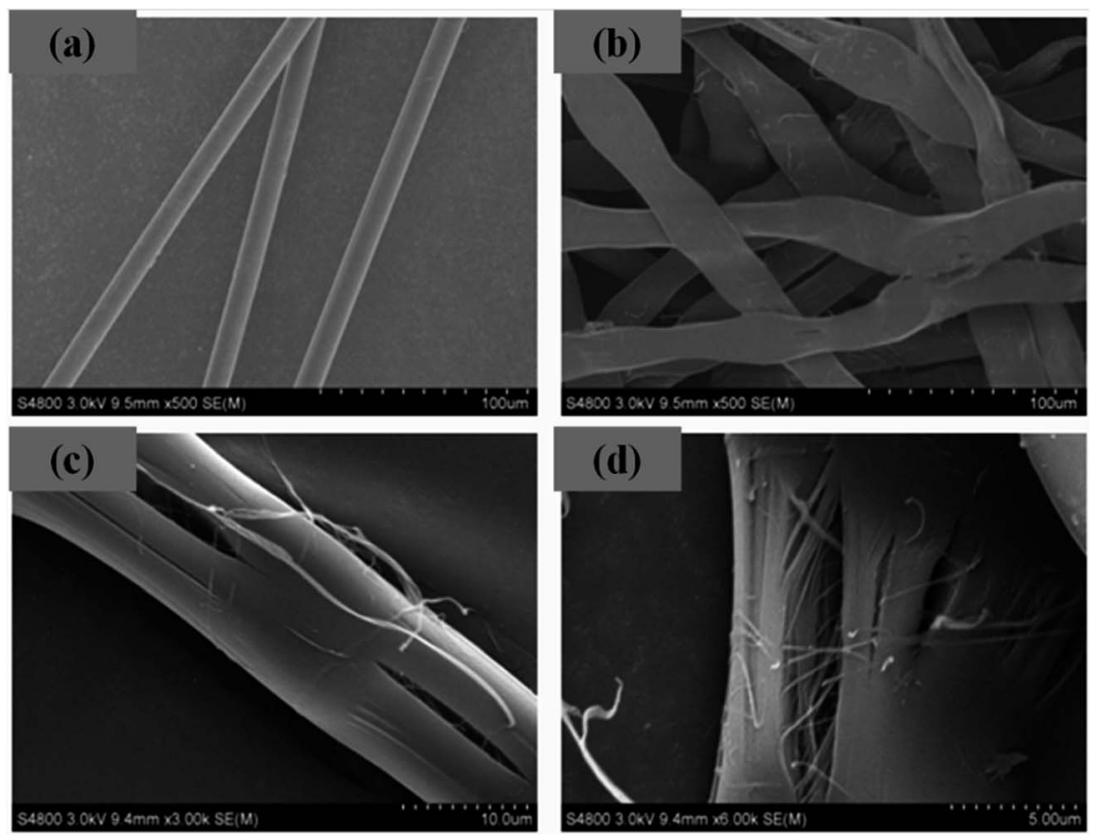

Fig. 10 Flattening, partial dissociation and partial-fibrillation process for the aramid chopped fiber under the combined heating/pressing treatment with increasing pressure: (a) the pristine aramid chopped fiber; (b) flattened fiber; (c) partial dissociated fiber; (d) partial fibrillated fiber.

fibrillation process. These fibrils are thus highly effective for mechanical reinforcement. As a result, partial fibrillar structure induced by the combined heating/pressing treatment is in great favor of the resultant mechanical property.

Fig. 11 summarized the paper structural properties of paraaramid fiber/fibrid composite papers. Two important components, namely, fiber and fibrid, have experienced great changes in morphologies and chemical structures due to hot-pressing especially the combined heating/pressing treatment. It was found that low pressure along with heating treatment induces flattening process, leading to strips of para-aramid fibers with smooth surface. Increased pressure can gradually induce partial dissociation and even partial fibrillation, indicating a high aspect ratio and greatly improved surface activity of aramid fibers. At the same time, fibrids can achieve high surface activity after hot-pressing, thus giving rise to an enhanced interfacial adhesion between aramid fibers and fibrids. Hence, Paper-II can finally achieve a super high mechanical property after
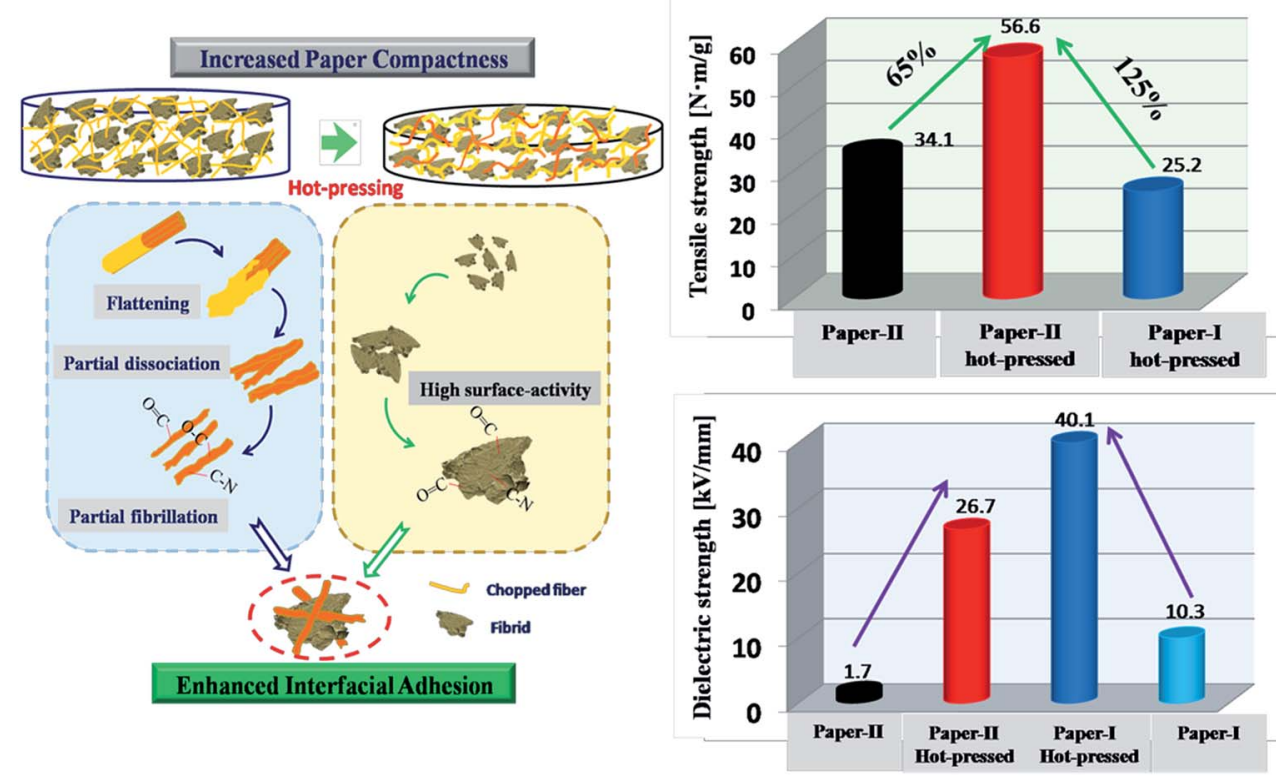

Fig. 11 Schematic profile for improving the overall physical properties of PPTA-based paper with the aid of hot-pressing process. 
hot-pressing. Nevertheless, the existence of pores and defects are bad for the insulation properties. As a result, the dielectric strength of Paper-I is higher than that of Paper-II. With the help of hot-pressing, paper compactness is remarkably increased and thus results in a further increase of insulation properties for both Paper-I and Paper-II. Therefore, Paper-II achieves a comprehensive improvement in mechanical properties as well as insulation properties.

\section{Conclusion}

In summary, the effect of hot-pressing on mechanical properties and insulating properties of para-aramid-based composite papers were studied. The results showed that hot-pressing is in great favor for enhancement of mechanical properties and insulation properties for both Paper-I and Paper-II. The mechanical properties and insulation properties are independent on individual heating treatment and are mainly dominated by individual pressing treatment. Meanwhile, the combined treatment of heating and pressing caused a synergistic effect, namely, further increasing the mechanical properties and insulation properties for both papers especially for Paper-II. Furthermore, the combined treatment also resulted in an increase of the paper compactness and the interfacial interaction between different fibers, all of which are mainly attributed to the flattening and even partial dissociation into fibrillar structure of para-aramid chopped fibers, and thus giving rise to enhanced surface activation, improved internal bonding and optimized mechanical and insulation properties for para-aramid fiber-based papers.

\section{Acknowledgements}

The authors would like to acknowledge the financial support from the National Key Research and Development Plan (2016YFB0303304), State Key Laboratory of Pulp and Paper Engineering (201333), the National Natural Science Foundation of China (Project: 51402180).

\section{References}

1 J. Gu, C. Liang, X. Zhao, B. Gan, H. Qiu, Y. Guo, X. Yang, Q. Zhang and D. Y. Wang, Compos. Sci. Technol., 2017, 139, 83-89.

2 Y. Zhao, Y. Zhu, G. Sui, F. Chen and Q. Fu, Polymer, 2017, 109, 137-145.

3 J. Gu, X. Yang, C. Li and K. Kou, Ind. Eng. Chem. Res., 2016, 55, 10941-10946.

4 J. Kratz and P. Hubert, Composites, Part A, 2015, 72, 219-227. 5 N. Ni, Y. Wen, D. He, X. Yi, T. Zhang and Y. Xu, Composites, Part B, 2016, DOI: 10.1016/j.compositesb.2016.01.042.

6 J. Gu, X. Yang, Z. Lv, N. Li, C. Liang and Q. Zhang, Int. J. Heat Mass Transfer, 2016, 92, 15-22.

7 J. Gu, X. Meng, Y. Tang, Y. Li, Q. Zhuang and J. Kong, Composites, Part A, 2017, 92, 27-32.

8 H. F. Zhao, M. Y. Zhang, S. F. Zhang and J. B. Lu, Polym.Plast. Technol. Eng., 2012, 51, 134-139.
9 S. Hayashida, in High-Performance and Specialty Fibers: Concepts, Technology and Modern Applications of Man-Made Fibers for the Future, ed. S. The Society of Fiber and J. Techno, Springer, Japan, Tokyo, 2016, pp. 149-169.

10 H. A. Every, G. J. M. Janssen, E. F. Sitters, E. Mendes and S. J. Picken, J. Power Sources, 2006, 162, 380-387.

11 L. R. Yao, W. T. Zhao, S. Q. Xu and Q. L. Sum, in Silk, Protective Clothing and Eco-Textiles, ed. L. Bai and G. Q. Chen, Trans Tech Publications Ltd, Stafa-Zurich, 2013, vol. 796, pp. 290-293.

12 P. Gopal, L. R. Dharani and F. D. Blum, Wear, 1996, 193, 199206.

13 M. Grujicic, W. C. Bell, P. S. Glomski, B. Pandurangan, C.-F. Yen and B. A. Cheeseman, J. Mater. Eng. Perform., 2011, 20, 1401-1413.

14 J. A. Mohandesi, A. Sangghaleh, A. Nazari and N. Pourjavad, Comput. Mater. Sci., 2011, 50, 1619-1624.

15 J. Gu, N. Li, L. Tian, Z. Lv and Q. Zhang, RSC Adv., 2015, 5, 36334-36339.

16 B. Yang, Z. Q. Lu, M. Y. Zhang, Y. J. Liu and G. D. Liu, J. Appl. Polym. Sci., 2016, 133, 43209-43215.

17 J. Fan, Z. Shi, M. Tian and J. Yin, RSC Adv., 2013, 3, 1766417667.

18 X. Li, F. Tian, P. Zhou, C. Yang, X. Li, F. Bian and J. Wang, RSC Adv., 2016, 6, 81552-81558.

19 L. Yao, K. Kim and J. Kim, Fibers Polym., 2012, 13, 277281.

20 J. K. Wan, H. Y. Lee, B. Baek and S. C. Yong, Text. Sci. Eng., 2015, 52, 26-34.

21 H. F. Zhao, Y. B. Zhu and L. Z. Sha, e-Polymers, 2014, 14, 351362.

22 S. F. Zhang, M. Y. Zhang and K. C. Li, Appita J., 2010, 63, 5864.

23 S. Zhang, M. Zhang and K. Li, Poly. Bull., 2011, 66, 351-362.

24 H. Song, Y. Wang, G. L. Xu and J. Hu, Preparation of phenolic resin for strengthening aramid paper, CRC Press-Taylor \& Francis Group, Boca Raton, 2015, vol. 42, pp. 5937-5946.

25 Z. Sun, S. Shi, X. Hu, X. Guo, J. Chen and H. Chen, Composites, Part B, 2015, 77, 38-45.

26 J. Gu, C. Liang, J. Dang, W. Dong and Q. Zhang, RSC Adv., 2016, 6, 35809-35814.

27 Y. Rao, A. J. Waddon and R. J. Farris, Polymer, 2001, 42, 59375946.

28 C. Li, Q. Zhen, Z. Luo and S. J. Lu, J. Appl. Polym. Sci., 2015, 132, 307-315.

29 S. Yang, L. Li, T. Xiao, D. Zheng and Y. Zhang, Appl. Surf. Sci., 2016, 383, 142-150.

30 A. Castro-Muñiz, F. Suárez-García, A. Martínez-Alonso and J. M. D. Tascón, J. Colloid Interface Sci., 2011, 361, 307-315.

31 L. X. Xing, L. Liu, Y. D. Huang, D. W. Jiang, B. Jiang and J. M. He, Composites, Part B, 2015, 69, 50-57.

32 Y. Y. Chu, X. G. Chen, D. W. Sheel and J. L. Hodgkinson, Text. Res. J., 2014, 84, 1288-1297.

33 C. X. Jia, P. Chen, Q. Wang, J. Wang, X. H. Xiong and K. M. Ma, Polym. Compos., 2016, 37, 620-626. 
34 M. Pykönen, H. Sundqvist, J. Järnström, O.-V. Kaukoniemi, M. Tuominen, J. Lahti, J. Peltonen, P. Fardim and M. Toivakka, Appl. Surf. Sci., 2008, 255, 3217-3229.

35 C. X. Wang, M. Du, J. C. Lv, Q. Q. Zhou, Y. Ren, G. L. Liu, D. W. Gao and L. M. Jin, Appl. Surf. Sci., 2015, 349, 333342.

36 X. Qian, R. Zou, Q. OuYang, X. Wang and Y. Zhang, Appl. Surf. Sci., 2015, 327, 246-252.

37 L. Luo, Y. Wang, J. Huang, D. Hong, X. Wang and X. Liu, RSC Adv., 2016, 6, 62695-62704.

38 Z. Cheng, P. Wu, B. Li, T. Chen, Y. Liu, M. Ren, Z. Wang, W. Lai, X. Wang and X. Liu, Appl. Surf. Sci., 2016, 384, 480486.

39 H. Zhang, J. Zhang, J. Chen, X. Hao, S. Wang, X. Feng and Y. Guo, Polym. Degrad. Stab., 2006, 91, 2761-2767.

40 Y. Y. Sun, Q. Liang, H. J. Chi, Y. J. Zhang, Y. Shi, D. N. Fang and F. X. Li, Fibers Polym., 2014, 15, 1-7.

41 J. Gao, Y. Y. Dai, X. Wang, J. Y. Huang, J. Yao, J. Yang and X. Y. Liu, Appl. Surf. Sci., 2013, 270, 627-633.

42 L. Z. Sha and H. F. Zhao, Polymer, 2013, 37, 196-203.

43 Z. Jia, Fibers Polym., 2013, 14, 59-64.
44 J. H. Kim, N. Alan Heckert, S. D. Leigh, R. L. Rhorer, H. Kobayashi, W. G. McDonough, K. D. Rice and G. A. Holmes, Compos. Sci. Technol., 2014, 98, 93-99.

45 Z. Cheng, B. Li, J. Huang, T. Chen, Y. Liu, X. Wang and X. Liu, Mater. Des., 2016, 106, 216-225.

46 L. Luo, P. Wu, Z. Cheng, D. Hong, B. Li, X. Wang and X. Liu, J. Fluorine Chem., 2016, 186, 12-18.

47 Z. Cheng, B. Y. Li, J. Y. Huang, T. Chen, Y. Liu, X. Wang and X. Y. Liu, Mater. Des., 2016, 106, 216-225.

48 J. Lim, J. Q. Zheng, K. Masters and W. W. Chen, International Journal of Impact Engineering, 2011, 38, 219-227.

49 D. B. Warheit, K. L. Reed, K. E. Pinkerton and T. R. Webb, Toxicol. Lett., 2002, 127, 259-267.

50 Z. Lu, M. Jiang, M. Zhang and X. Xia, J. Eng. Fibers Fabr., 2016, 11, 1-8.

51 Y. Sun, Y. Wang, G. Xu, F. Cheng and J. Hu, Text. Res. J., 2016, 86, 1533-1542.

52 J. M. Zhang, Z. Mousavi, N. Soykeabkaew, P. Smith, T. Nishino and T. Peijs, ACS Appl. Mater. Interfaces, 2010, 2, 919-92653.

53 W. Zhai, H. Liu, Z. Hong, W. Xie and B. Wei, Ultrason. Sonochem., 2017, 34, 130-135. 